\title{
Verbal insight revisited: fMRI evidence for early processing in bilateral insulae for solutions with AHA! experience shortly after trial onset
}

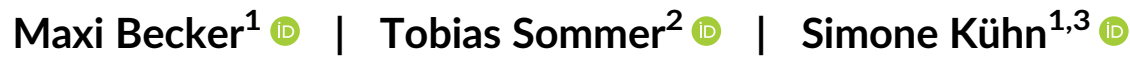

${ }^{1}$ Clinic and Policlinic for Psychiatry and Psychotherapy, University Medical Centre Hamburg-Eppendorf, Hamburg, Germany

${ }^{2}$ Department of Systems Neuroscience, Neurolmage Nord, University Medical Centre Hamburg-Eppendorf, Hamburg, Germany

${ }^{3}$ Center for Lifespan Psychology, Max Planck Institute for Human Development, Berlin, Germany

\section{Correspondence}

Maxi Becker, Clinic and Policlinic for Psychiatry and Psychotherapy, University Medical Centre Hamburg-Eppendorf, Martinistraße 52, Hamburg 20246, Germany. Email: maxi_becker@gmx.net

Funding information

European Research Council, Grant/Award Number: ERC-2016-StG-Self-Control-677804; German Science Foundation, Grant/Award Numbers: DFG KU 3322/1-1, SFB 936/C7

\begin{abstract}
In insight problem solving solutions with AHA! experience have been assumed to be the consequence of restructuring of a problem which usually takes place shortly before the solution. However, evidence from priming studies suggests that solutions with AHA! are not spontaneously generated during the solution process but already relate to prior subliminal processing. We test this hypothesis by conducting an fMRI study using a modified compound remote associates paradigm which incorporates semantic priming. We observe stronger brain activity in bilateral anterior insulae already shortly after trial onset in problems that were later solved with than without AHA!. This early activity was independent of semantic priming but may be related to other lexical properties of attended words helping to reduce the amount of solutions to look for. In contrast, there was more brain activity in bilateral anterior insulae during solutions that were solved without than with AHA!. This timing (after trial start/during solution) $x$ solution experience (with/without $A H A$ !) interaction was significant. The results suggest that (a) solutions accompanied with AHA! relate to early solutionrelevant processing and (b) both solution experiences differ in timing when solution-relevant processing takes place. In this context, we discuss the potential role of the anterior insula as part of the salience network involved in problem solving by allocating attentional resources.

KEYWORDS

aha experience, CRA, fMRI, insight, insula, priming
\end{abstract}

\section{1 | INTRODUCTION}

In this study, we investigate the physiological basis of the subjective feeling of insight-the AHA! Experience in compound remote associates (CRAs). In particular, we explore how the solution process differs between problems that are solved with and without AHA! experience by focusing not only on the end, but also on the beginning of the solution process and the search for a solution.

\section{1 | CRAs as a means to study the AHA! experience}

A task that has been widely used to study insight are CRA problems which are an extended version of the classic Remote Associate Test originally developed by Mednick (1962) (Bowden, Jung-Beeman, Fleck, \& Kounios, 2005; Kounios \& Beeman, 2014). CRA problems consist of three target words (pine, pie, sauce) and the task of the problem solver is to find the one compound word (apple because pineapple, apple pie, and applesauce) that represents a meaningful compound to each of the three target words (Bowden \& Jung-Beeman, 2003a, 2007). It is assumed that solution-irrelevant associations of the target words (pine as in pine tree) are often activated first in CRAs (Bowden \& Jung-Beeman, 2003a). For this reason, the solution does not immediately reach awareness. As a consequence, the solution seems nonobvious and remote which is why the subjects often start searching for possible solutions. However, when finding the solution 
word, it is often accompanied by an AHA! experience of insight. Here, we define insight as a sudden comprehension or solution of a nonobvious problem that involves an AHA! experience (note, there are various definitions of insight but we concentrate on the AHA! experience, Kounios \& Beeman, 2014). The subjective feeling of an insight-the AHA! experience-has been associated with sudden ease, the strong belief that the solution is true and sometimes a feeling of pleasure (Topolinski \& Reber, 2010). In contrast, CRA problems can also be solved without the above-mentioned properties (without AHA! experience)-for example via trial-and-error. Indeed, solved problems that are accompanied with an AHA! experience are more accurate than solutions without this subjective feeling of AHA! (Salvi, Bricolo, Franconeri, Kounios, \& Beeman, 2015). But what causes an AHA! experience and why do both solution experiences (with/without AHA!) differ?

\section{2 | Explanations for the AHA! experience}

It has long been assumed that solutions with an AHA! experience occur when the problem solver initially focuses on solution-irrelevant associations (pine as in pine tree)-eventually gets stuck-but manage to retrieve the correct solution word from memory by switching the focus to solution-relevant associations (pine as in pine apple) (Bowden \& Jung-Beeman, 2003a; Kounios \& Beeman, 2014; Subramaniam, Kounios, Parrish, \& Jung-Beeman, 2009). This process of switching the attentional focus to solution-relevant problem elements and thereby changing the problem presentation has also been referred to as restructuring (e.g., Knoblich, Ohlsson, Haider, \& Rhenius, 1999; Kounios \& Beeman, 2014; Ohlsson, 1992). There is a lot of evidence from classic insight tasks (nine-dot or eight-coin problem), that the AHA! experience follows successful restructuring (Öllinger, Jones, \& Knoblich, 2008; Öllinger, Jones, \& Knoblich, 2014; Thevenot \& Oakhill, 2008). However, recent studies demonstrate that restructuring can lower the likelihood of an AHA! experience (Becker, Wiedemann, \& Kühn, 2018) or even take place in the absence of an AHA! (Danek, Wiley \& Öllinger, 2016). In contrast, when people solve anagrams, they experience their solutions more insight-like when solution-related words were presented to them subliminally prior to solution (Bowden, 1997). Furthermore, subjects were more likely to rate their CRA solution with an AHA! experience when they had prior semantic activation of the solution, as indexed by solution priming (Bowden \& Jung-Beeman, 2003b).

These findings suggest two critical aspects: On the one hand, the AHA! experience may not be causally related to restructuring of a problem. On the other hand, the fact that semantic priming can influence later solutions accompanied by an AHA! supports the hypothesis that solutions with $\mathrm{AHA}$ ! experience relate to prior subliminal processing rather than being spontaneously generated (see, Kounios \& Beeman, 2014). Subliminal processing refers to any solution-relevant mental computation that is below the solver's threshold of awareness. Bowden and Jung-Beeman assume that solution-related associations in problems later solved with $\mathrm{AHA}$ ! are activated prior to the solution but below the threshold of awareness or overshadowed by other solution-irrelevant activations (Bowden \& Jung-Beeman, 2003b). Finally, this would imply that solutions with and without AHA! experience already differ in their cognitive process before the actual problem is restructured and subsequently solved, at least in verbal open problems like CRAs or anagrams. At the same time, it is unknown whether the increased likelihood of a solution accompanied by an AHA! in the above-mentioned priming studies is only due to a potential priming influence automatically activating semantically related associations to the solution word shortly after problem presentation or whether early processing of problems solved with an AHA! is independent of priming.

\subsection{Neural basis for the AHA! experience}

There has been substantial research in the last decade investigating the neural basis for solutions accompanied with an AHA! in insight problems (see Shen, 2018 for a meta-analysis). Increased activations in the medial temporal lobe (amygdala, hippocampus, and parahippocampal gyrus) (Jung-Beeman et al., 2004; Kizilirmak, Thuerich, Folta-Schoofs, Schott, \& Richardson-Klavehn, 2016; Ludmer, Dudai, \& Rubin, 2011; Zhao et al., 2013) and right anterior superior temporal gyrus (Jung-Beeman et al., 2004; Tik et al., 2018) amongst other areas have been reported in solutions with compared to without AHA! experience. The right anterior superior temporal gyrus has been associated with integration of information across distant semantic relations (Jung-Beeman et al., 2004); the hippocampus has been linked to formation of novel associations (Luo \& Niki, 2003) and the amygdala was associated with positive emotional response to sudden comprehension (Ludmer et al., 2011). Furthermore, heightened activity in medial frontal areas associated with cognitive control, for example, the anterior cingulate cortex (ACC) before the respective problem is presented has been associated to facilitate solutions with an AHA! experience (Kounios et al., 2006; Subramaniam et al., 2009).

However, most research investigating the AHA! experience reports brain data at the time of solution or a few seconds before (e.g., Jung-Beeman et al., 2004; Kizilirmak et al., 2016; Luo, Niki, \& Phillips, 2004; Tik et al., 2018; Zhao et al., 2013), for the whole trial (Tik et al., 2018) or before the respective trial starts (Kounios et al., 2006; Subramaniam et al., 2009) but not shortly after trial presentation or during the time of search for the solution compound. Only Aziz-Zadeh, Kaplan, and lacoboni (2009) investigated neural correlates of anagram solutions directly after trial start using fMRI. Within the first $3 \mathrm{~s}$ after problem presentation, the authors report stronger bilateral insula and left inferior frontal gyrus activation for anagram problems that were later reported to be solved with than without an AHA! experience (Aziz-Zadeh et al., 2009). Although the authors do not relate their results to time-related differences between both solution experiences, this suggests that solutions are differentially processed already shortly after problem presentation as a function of the solution experience. However, it is unclear whether these results also generalize to CRAs and whether priming influences early solution processing. Furthermore, the authors did not compare this activation to the time when the participants had found the solution. Comparing 
brain activation after task start, while searching for and when having found the solution could present a more detailed picture of how problems that were later solved with or without an AHA! are processed in the brain (Sandkühler \& Bhattacharya, 2008).

\section{4 | Hypotheses}

Given the above-mentioned evidence, we hypothesize that both solution experiences (with/out AHA!) differ according to how early solution-relevant processing takes place. Thus, problems later solved with an AHA! experience are already processed early (shortly after trial start) and thus before reaching the solution. Here, we define solution-relevant processing as every task-related mental operation that facilitates the process of finding the correct solution word (this term does not specify whether this mental operation is initiated willingly by the solver or happens subliminally, that is, below the threshold of the solvers awareness).

If solution-relevant processing is initiated early, retrieving the solution word should require less conscious effort for problems solved with than without AHA! experience. In particular, we hypothesize the following:

In line with the results of Aziz-Zadeh et al. (2009), we expect more solution-relevant processing already shortly after trial start. Therefore, we expect brain activation in frontal areas like the left IFG and insula within the first $3 \mathrm{~s}$ after CRA problem presentation for solutions with than without AHA! experience.

In line with prior research, we assume an increased likelihood for solutions accompanied with an AHA! experience when a semantically related compared to an unrelated prime is presented together with the problem (Bowden, 1997; Bowden \& Jung-Beeman, 2003b). However, since there is no prior $\mathrm{fMRI}$ evidence for the influence of semantic priming on CRA problems that are later solved with an AHA! experience directly after trial start, two scenarios are possible: First, the semantically related prime affects problem solution already after trial start in those areas that show differential blood oxygen level dependent (BOLD) activation between solutions with and without AHA! experience. In this case, the AHA! experience could be explained by the related prime activating solution-relevant associations which then could be more easily retrieved from memory (Bowden \& Jung-Beeman, 2003b). Second, there is no priming effect within the first $3 \mathrm{~s}$ after task start but only during later solution phases. In this second scenario, early solution-relevant processing of problems later solved with AHA! is independent of semantic priming (prior activation of solution-relevant associations).

If solution-relevant processing is initiated early, we would also assume that problems solved with compared to without an AHA experience take less solution time and are solved with less search effort and search attempts. Similarly, we expect more cognitive control areas to be activated in solutions without than with AHA! experience during search and solution indicating retrieval of the solution word to be more effortful.

In order to test these hypotheses and the possible influence of semantic priming on the $\mathrm{AHA}$ ! experience during early problem processing, we analyzed data from an fMRI study using a modified CRA paradigm adopting semantic priming (see, Becker et al., 2018). That is to say, a prime that is either semantically related or unrelated is additionally presented with the CRA item. This design allows to differentiate the solution experience (with/out AHA!) from the influence of the prime as well as different solution phases (trial start, search, and solution).

In addition, we conducted an independent control experiment using eye tracking with the same experimental set-up. This control study enabled us to further investigate which lexical properties next to semantic distance to the solution may relate to early solutionrelevant processing and predict later solutions with an AHA! experience.

\section{2 | MATERIALS AND METHODS}

\section{1 | Participants}

For the fMRI study, we included 30 healthy right-handed participants recruited via an online student platform in Hamburg (age [in years]: range $=18-31,23$ females: $M=23.5 ; 7$ males: $M=25.1$ ). For the eye tracking control study, we included 26 healthy participants recruited via the same online student platform in Hamburg (age [in years]: range $=20-35,23$ females: $M=25.7 ; 7$ males: $M=26.9$ ). All participants had normal or corrected-to-normal vision, were German native speakers and received a financial compensation for their participation. The ethics committee of the German society for psychology approved of this study. Informed consent was obtained from all individual participants included in the study. We selected the participants based on their performance (at least $40 \%$ accuracy) in an online pretest. In this online test, subjects were asked to solve 16 CRAs. Due to lower performance than $40 \%$ accuracy in the pretest, 14 (3) participants were rejected from the fMRI (eye tracking) study prior to invitation. This procedure was necessary to ensure that the invited subjects would most likely produce a sufficient number of events for later analyses. To ensure similar conditions for both studies, we kept the same cutoff criterion (40\% accuracy) for the eye tracking study. For the fMRI analyses, we had to exclude three from the included 30 participants because they either did not press any search buttons (see Section 2.2) or they indicated to only have solved the CRA problems without AHA! experience.

\section{2 | Experimental design}

We used a modified CRA task including semantic priming as described in detail in Becker et al. (2018): First, a prime (dew or back) that is semantically more or less related to the solution is presented followed by three target words (drop, coat, proof) (see, Figure 1). The goal is to find the compound word that builds a meaningful compound with every one of the target words and can be appended in front or in the back of each one of them. In addition, the presented prime also builds a meaningful compound with the first target word changing its meaning to be more closely related to the solution compound (dewdrop 
FIGURE 1 Example of modified CRA paradigm. Note. (a) dew: example for semantically related and (b) back: for unrelated prime to solution (rain)

\begin{tabular}{|c||c|}
\hline $\begin{array}{c}\text { Related prime (RP) } \\
\text { (a) DEW: DROP - COAT - PROOF }\end{array}$ & (a) DEW \\
\hline $\begin{array}{c}\text { Unrelated prime (UP) } \\
\text { (b) BACK: DROP - COAT - PROOF }\end{array}$ & (b) BACK $-\cdots$ \\
\hline
\end{tabular}

vs. raindrop) or to be more semantically distant to it (backdrop vs. raindrop; see, Figure 1). Hence, there is a twofold relationship between the prime/compound and the solution/compound (dew, back to rain as well as dewdrop, backdrop to raindrop). We assumed that the twofold semantic relationship between the prime/compound and the solution/compound increases the amount of solution ir/relevant associations in the participants.

We utilized 66 modified CRA items in German language as published in Becker et al. (2018). The items were validated according to their lexical (word frequency, word length) and statistical properties (accuracy, solution time). To ensure that the respective primes (or prime compounds) were semantically related to the solution (or solution compound), the authors estimated the semantic distance computationally via statistical cooccurrences in text data using a word embedding model. The semantic relationship between two word pairs (e.g., dew, rain, target word and solution word) was estimated by measuring the cosine distance between both word vectors (the method of computationally estimating the semantic distance via cosine distance is described in detail in Becker et al., 2018 and will be omitted here for lack of space). The average cosine distance between the semantically related prime and the solution is $M=.55(S D=.14)$ and between the unrelated prime and the solution is $M=.72(S D=.07)$. This difference in cosine distance is significant between both conditions $(t[65]=-8.57, p<.001)$. The value range of the cosine distance is between 0 and 1 . A value close to 1 is interpreted as very high semantic distance (little semantic similarity). In addition, the difference in cosine distance between the related $(M=.41, S D=.08)$ and unrelated $(M=.59, S D=.09)$ prime compound to the solution compound is also statistically significant $(t[65]=-11.8, p<.001)$.

Isolating the differential effects of the semantic distance between the prime/compound and solution/compound on behavior and the BOLD response during search and solution using this paradigm is beyond the scope of the present manuscript but reported elsewhere (Becker, Sommer, \& Kühn, in press; Becker et al., 2018).

\section{3 | Procedure}

Before entering the scanner, participants had to complete four test trials of the modified CRA items to ensure that they understood the task and complied with the task instructions. They were orally instructed that the solution word is a common word from the German dictionary and could be put in front or in the back of each of the target words. Additionally, they were told that the prime can but does not have to be semantically related to the solution word. A solution with and without AHA! experience was explained to them as follows:

\begin{abstract}
"The defining characteristic of a solution with an AHA! experience is how sudden and obviously correct the solution appears to you. This can also be the case when you have already searched for the solution for quite some time. In contrast, the solution without AHA! appears to you more in a stepwise manner and not sudden. For example, through active search you feel like you increasingly approached the solution."
\end{abstract}

The participants held a button press device with five response options in their right hand. They were instructed to press Button 1 when they found the final solution and Button 2 for all possible but wrong solutions that came to their mind (search). The subjects received max. 66 items $(M=60, S D=6)$ in three consecutive blocks each lasting max. $15 \mathrm{~min}$. The session order and the prime conditions were counter balanced between all subjects. The modified CRA items were presented for maximally 60 se in random order per session until time out.

The CRA problems were presented on a screen in the MRI, which participants watched via a mirror system. The sequence of the experiment was as indicated in Figure 2: After a short presentation of a fixation cross $(600 \mathrm{~ms})$ announcing the new trial, the prime word appeared on the screen for $2 \mathrm{~s}$ followed by the three target words next to the prime. The prime remained on the screen together with the target words for max. $60 \mathrm{~s}$ or until the solution button (Button 1) was pressed. Note, the effect of the prime on semantic processing can therefore take place at different time points and is less strongly controlled. However, the continuous presentation of the prime was chosen to ensure that participants actively represent the solution relevant (dewdrop) or irrelevant meaning (backdrop) of the first target word as a function of the respective prime for the entire time. If participants exceeded those $60 \mathrm{~s}$ without pressing the solution button (Button 1), a new fixation cross (600 ms) would appear followed by a question how much effort they invested in finding a solution (range: 1-5). Upon response a new trial would begin. While searching for the solution, participants were instructed to press Button 2 every time they came up with a possible but wrong solution.

If they did press Button 1 (subjective final solution), they received five response options for $8 \mathrm{~s}$ with four gap words (e.g., $\mathrm{R}_{-} \mathrm{-}_{\mathrm{N}} \mathrm{N}$ ) and one question mark indicating an alternative solution. The four gap words entailed the correct solution ( $\mathrm{R}_{-} \mathrm{N}_{\mathrm{N}}$ for rain) and three incorrect solution words (e.g., S _ _ W, T _ _ L, E _ _ R). Because participants could not type their correct answer in the scanner they had to choose one of the options. The reason for the short decision period of $8 \mathrm{~s}$ was to prohibit participants from reevaluating their solution using the gap words. Participants responded to have found alternative solutions 


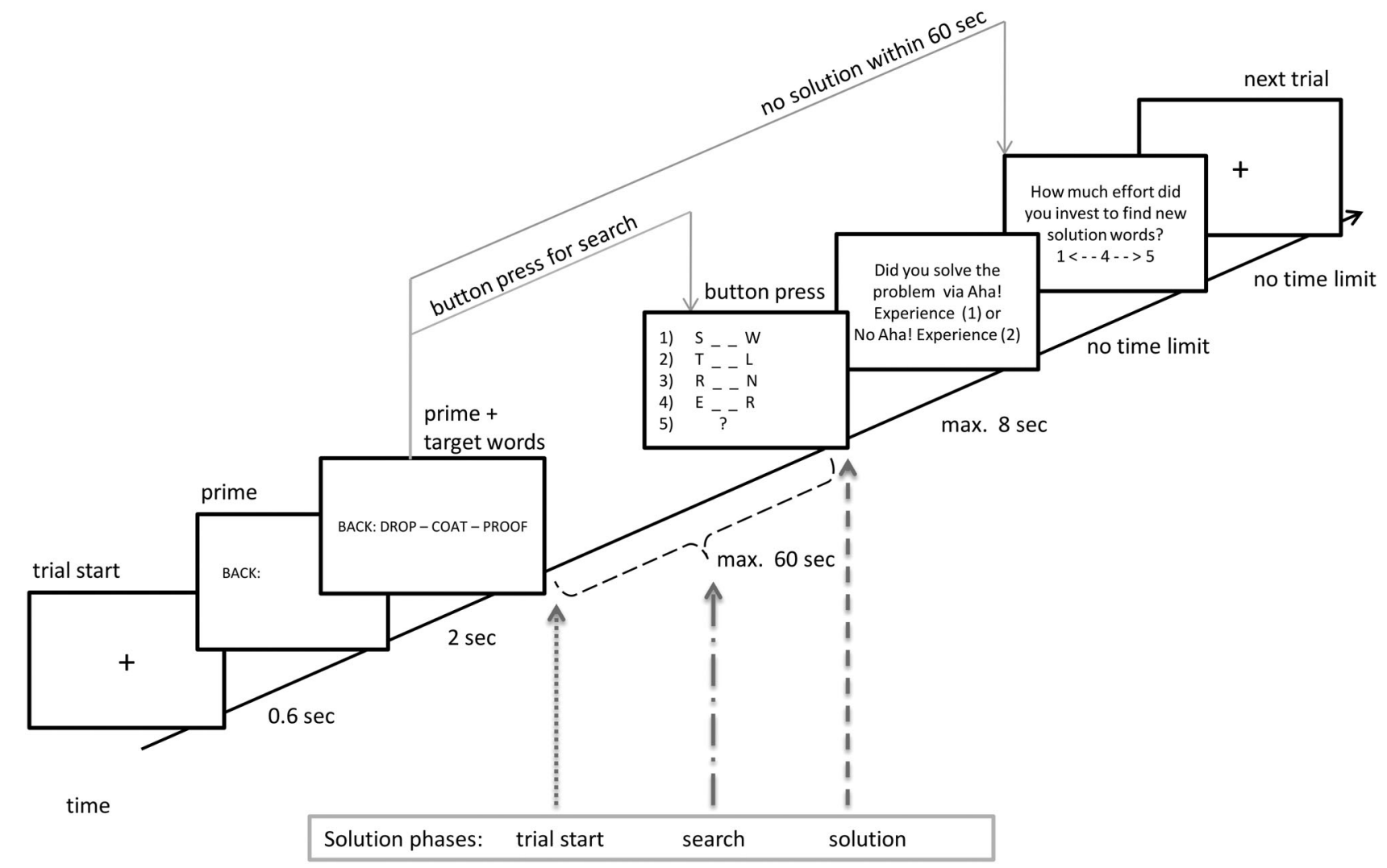

FIGURE 2 Time flow of the modified CRA paradigm and solution phases. Note. The subjects were instructed to press the search button (2) every time they thought of a possible but wrong solution and another button (1) when they found the final solution. If the subjects indicated to have found the solution, they were asked whether the solution was found with or without AHA! experience. Three solution phases were differentiated for the fMRI analyses: (a) the first $3 \mathrm{~s}$ after trial start, (b) the onset of every search button press (2) and (c) the onset of every final solution button press (1)

(a solution that did not fit to either of the four presented gap words) in $7.5 \%$ of all cases. Because we cannot verify whether these solutions are correct, we discarded those answers for the calculation of accuracy and from all fMRI analyses. Finally, participants were asked whether they had an AHA! experience when solving the problem or not. This response was followed by a question about their search effort and subsequently a new trial would start (see, Figure 2).

\section{4 | Behavioral data analysis}

We modeled the behavioral responses from the fMRI experiment using a generalized linear mixed model (GLMM; Baayen, Davidson, \& Bates, 2008). Our dependent variable of interest was whether participants indicated to have found the solution accompanied by an AHA! experience or without it (binary variable).

Accuracy (item in/correctly solved-binary variable), solution time (reaction time to solution per item-continuous variable), semantic priming (semantically related or unrelated prime-binary variable) as well as amount of search (amount of search button presses per trialcount variable) and perceived effort to have found the solution (continuous variable between 1 and 5) served as independent variables. For control purposes, we checked whether sex is a significant predictor for likelihood to report an AHA! experience given that we included $\sim 77 \%$ females into the study but we found no evidence supporting this $(p>$.7). For this reason, the GLMM will be reported without sex as independent predictor.

We modeled all subjects and CRA items as random intercept effects and carried out the analyses using R (R Core Team, 2014) and the Ime4 package (Bates, Maechler, Bolker, \& Walker, 2014). The AHA! experience variable was modeled assuming a binomial error distribution with the default logit link function (Bates et al., 2014). We calculated a GLMM with accuracy, solution time, search, and search effort and un/related prime as fixed effects. The output summary and the $p$-values for the single predictors were obtained via the sjPlotpackage in $\mathrm{R}$ (Lüdecke, 2018).

\section{5 | MRI data acquisition, preprocessing, and statistical fMRI analysis}

Brain images were collected on a 3 Tesla Siemens Magnetom Skyra MRI scanner system (Siemens Medical Systems, Erlangen, Germany) using a 20-channel radiofrequency head coil. The structural images were collected using a three-dimensional T1-weighted magnetization prepared gradientecho sequence (MPRAGE) (repetition time $(T R)=2,500 \mathrm{~ms}$; echo time $(\mathrm{TE})=2.12 \mathrm{~ms} ; \mathrm{TI}=1,100 \mathrm{~ms}$, acquisition matrix $=256 \times 256 \times 192$, flip angle $=9^{\circ} ; \mathrm{FOV}=240 \mathrm{~mm}$, voxel size $=0.8 \mathrm{~mm} \times 0.8 \mathrm{~mm} \times 0.9 \mathrm{~mm}$ ). 
TABLE 1 Representation of modeled events throughout the solution process for $\mathrm{fMRI}$ analysis

\begin{tabular}{|lll}
\hline Solution phase & Event type & Description \\
\hline Trial start & CRA items presented with: \\
\hline & $\begin{array}{l}\text { 1. Related_Prime_AHA } \\
\text { 2. Unrelated_Prime_AHA }\end{array}$ & Related prime later solved with AHA after trial start \\
\hline 3. Related_Prime_noAHA & Related prime later solved without AHA after trial start \\
\hline 4. Unrelated_Prime_noAHA & Unrelated prime later solved with AHA after trial start \\
\hline Search & Unrelated prime later solved without AHA after trial start \\
\hline Solution & 6. noAHA & \\
\hline & CRA items solved with AHA during search \\
\hline 8. Related_Prime_AHA & CRA items solved without AHA during search \\
\hline 9. Related_Prime_noAHA & CRA items presented with: \\
\hline 10. Unrelated_Prime_noAHA & Related prime later solved with AHA during solution \\
\hline
\end{tabular}

Functional images were obtained using a T2*-weighted echo planar imaging (EPI) sequence sensitive to BOLD contrast $(\mathrm{TR}=2,400 \mathrm{~ms}, \mathrm{TE}=30 \mathrm{~ms}$, image matrix $=64 \times 64$, FOV $=216 \mathrm{~mm}$, flip angle $=80^{\circ}$, voxel size $=3.0 \mathrm{~mm} \times 3.0 \mathrm{~mm} \times 3.0 \mathrm{~mm}, 36$ axial slices). Note, repetition time is $2,400 \mathrm{~ms}$ and not $2000 \mathrm{~ms}$ as in other studies investigating the AHA! experience (e.g., Jung-Beeman et al., 2004). The reason for this parameter choice was a trait-off due to the fact that we wanted to reach a whole brain coverage including the cerebellum.

\subsection{1 | Image processing}

We used SPM12 (Welcome Department of Cognitive Neurology, London, UK) to conduct all fMRI data analyses. The preprocessing pipeline was performed using the default SPM12 parameter choices. The imaging series was slice-time corrected, realigned, and coregistered to the individual structural image. Structural images were segmented into cerebral fluid and white and gray matter. Structural scans were further normalized to Montreal Neurological Institute (MNI) space and we used an 8-mm FWHM-Gaussian filter to smooth the functional images.

\subsection{2 | Statistical analyses}

First and second level analyses were conducted in the framework of general lineal models as implemented in SPM. For the first level analysis, we created a total of 20 separate regressors:

We modeled a) the first $3 \mathrm{~s}$ of each trial onset for problems that were either presented with a semantically related or unrelated prime split by whether these items were later solved with or without AHA! experience. Hence, for the trial onset we modeled four possible events (items with related prime and solved (1) with or (2) without AHA!, items with unrelated prime solved (3) with or (4) without AHA!). Note, the numbers 1-20 indicate the regressor numbers. For clarity, we abbreviated the four possible conditions (Related_Prime_AHA, Unrelated_Prime_AHA, Related_Prime_noAHA, Unrelated_Prime_noAHA) as shown in Table 1. We used the average BOLD signal of the first $3 \mathrm{~s}$ after problem presentation given the evidence for signal differences between both solution experiences as reported in Aziz-Zadeh et al. (2009). To ensure that this onset regressor is not confounded with search or solution button presses, we excluded all trials (10.66\%) where a search or solution button was pressed within these $3 \mathrm{~s}$ (however, excluding only solution button presses [0.79\%] from this onset regressor did not change the reported results).

We further modeled the onset of all the button presses for (b) the search phase (button press (2) for problems that were later solved (5) with or (6) without AHA! experience (see, Table 1). We did not further distinguish search events by splitting them up according to the presentation of related and unrelated prime items because this differentiation was not relevant to the research question.

Finally, we also modeled the onset of all solution button presses for problems that were presented with a related prime and solved (7) with or (8) without AHA! experience as well as problems that were presented with an unrelated prime and solved (9) with or (10) without AHA! experience (for an overview of all modeled events at each solution phase, see Table 1). The eleventh onset regressor was of no interest and included all other remaining button presses. These included for example button presses regarding one of the five response options. All onset regressors were convolved with a canonical hemodynamic response function (HRF) and its first temporal derivative to form the main regressors in the design matrix. Finally, we further included three regressors representing the mean for each of the three runs (regressors12-14) and six motions parameters (regressors 15-20). A high-pass filter with a $128 \mathrm{~s}$ cutoff period was applied to remove baseline drifts. Simple contrast images were calculated for each individual from the beta weights of every regressor containing the canonical HRF.

At the group level, we performed a random effects analysis to compare between the 10 different conditions as indicated in Table 1 (CRA items presented with un/related prime and their solutions accompanied with/out AHA!) at different phases (trial start, search, solution) during the task process. 
We used SPM's full factorial design entering the simple contrast images of the above-mentioned first 10 onset regressors (trial start with the four conditions: Related_Prime_AHA, Unrelated_Prime_AHA, Related_Prime_ noAHA; Unrelated_Prime_noAHA; search button presses of trials that were later solved with $\mathrm{AHA}$ ! and without $\mathrm{AHA}$ !; solution button presses with the four conditions: Related_Prime_AHA, Unrelated_Prime_AHA, Related_Prime_noAHA, Unrelated_Prime_noAHA).

Given our hypotheses, we contrasted the four conditions (Related_Prime_AHA, Unrelated_Prime_AHA, Related_Prime_noAHA, Unrelated_Prime_noAHA) after trial start and during solution. During search, we only contrasted items later solved with or without AHA! as we did not model the prime separately. The precise contrasts will be reported in Section 3. We excluded three participants from this group analysis $(n=27$ ) because they indicated less than 5 search button presses per condition (with/out AHA!) or did not indicate to have solved a problem accompanied by an AHA! experience.

To avoid confounding interindividual effects due to age (Old \& Naveh-Benjamin, 2008) and gender (77\% of our participants were female), we added these two variables as covariates of no interest into the full factorial design. However when individually modeling them with a simple contrast of 1 , no clusters were significant.

\subsection{3 | Conjunction and interaction analysis}

Because we observed bilateral insula activation during trial start $(\mathrm{AHA}>$ noAHA) and during solution (noAHA > AHA), we further investigated whether there was a solution experience $\times$ solution phase interaction. To explore whether there are commonly activated voxels in both contrasts and to assure that only those voxels were considered for the interaction analysis that show a main effect in the respective contrast, we first conducted a conjunction analysis of both contrasts $(\mathrm{AHA}>\mathrm{noAHA}$ during trial start and noAHA > AHA during solution). We then used the remaining significant clusters as mask to conduct a whole-brain interaction analysis with the contrast: (trial start: AHA - noAHA) - (solution: noAHA - AHA).

No differences in hemodynamic response due to the prime condition during trial start could be observed but only later during solution and only for solutions with an AHA! experience. To investigate whether the effect of the prime in the BOLD response for solutions with AHA! is related to the differences in BOLD contrast noAHA - AHA, we additionally conducted a conjunction analysis of the two contrasts (noAHA > AHA and Unrelated_Prime_AHA > Related_ Prime_AHA during solution). Both conjunction as well as interaction analyses were carried out for exploratory purposes.

The resulting statistical values from all whole-brain analyses were thresholded at a voxel level of $p<.001$ ( $z>3.09$, uncorrected). To correct for multiple comparisons, we adopted a cluster-level FWE correction at $p<.01$ for all reported whole-brain analyses (Eklund, Nichols, \& Knutsson, 2016). Only for the additional exploratory analyses-in particular the conjunction analysis (AHA > noAHA during trial start and noAHA > AHA during solution) and interaction analysis (trial start: AHA - noAHA) - (solution: noAHA - AHA)-we adopted a more lenient cluster-level FWE error correction of $p<.05$. All resulting statistical maps were superimposed on the averaged normalized structural image of all participants. Anatomical areas were determined using the AAL atlas (Tzourio-Mazoyer et al., 2002) based on the peak voxels from the random effects analysis. The reported coordinates correspond to the Montreal Neurological Institute (MNI) coordinate system (MNI; Evans et al., 1993).

\subsection{4 | Region of interest analysis}

For exploratory purposes, we additionally performed a region of interest (ROI)-based analysis in marsbar (Brett, Anton, Valabregue, \& Poline, 2002) for the contrast Related_Prime_AHA + Unrelated_Prime_AHA > Related_ Prime_noAHA + Unrelated_Prime_noAHA during solution. The wholebrain analysis for this contrast revealed one activation cluster in the left hippocampus as hypothesized, however, it did not reach significance $(p=.056$ ). To prevent double-dipping, we created a single broad mask by combining the anatomical regions of the hippocampus, amygdala, and parahippcampal gyrus from both hemispheres from the AAL atlas (TzourioMazoyer et al., 2002). Finally, we assumed a more lenient significance threshold of $p<.05$ for the $t$-test because there was no need to correct for multiple comparison.

\section{6 | Eyetracking control experiment, hypotheses, and data analysis}

Our motivation to conduct this control experiment was the following: Although the fMRI analysis revealed increased bilateral insula activation during the first $3 \mathrm{~s}$ after trial start for solutions with than without AHA! experience, this difference could not be explained by the semantically un/related prime. This was surprising given that we replicated the behavioral priming effect from earlier studies (Bowden, 1997; Bowden \& Jung-Beeman, 2003b). We therefore hypothesized that the early difference in brain activation as a function of the AHA! experience must be related to either the semantic relationship of one the three target words to the solution or to other lexical word properties of the presented words. In the style of earlier work, we identified (1) frequency and (2) amount of possible compounds that can be built with the respective word (we will refer to this as amount of compounds) and (3) semantic relationship of the respective word to the solution as relevant lexical properties (see, Becker et al., 2018).

Specifically, we hypothesized that lexical properties of the most highly attended word within the first $3 \mathrm{~s}$ after trial start predict the likelihood of a solution with an AHA! experience (note, this can be the prime or one of the target words). As a proxy for the most highly attended word, the amount of fixations per word within the first $3 \mathrm{~s}$ after trial start was counted. We therefore constructed regions of interest (rectangle) around each word (prime and each target word). Fixation events were identified using the commonly used parameter definitions (the EyeLink in its cognitive configuration uses velocity, acceleration, and motion thresholds of $30^{\circ} / \mathrm{s}, 8,000^{\circ} / \mathrm{s}^{2}$, and $0.15^{\circ}$, respectively (SR Research, 2007).

Eye tracking was done using an Eyelink 1000 Desktop Mount system (SR Research, ON, Canada) recording the right eye at 1,000 Hz. The CRA items were presented on a $20^{\prime \prime}$ monitor $(1,280 \times 960$ pixels, $60 \mathrm{~Hz})$ using 
Matlab2013a (Mathworks Natwick MA) with the psychophysics toolbox (Brainard, 1997). The distance of the participants' eyes to the stimulus presentation screen was $50 \mathrm{~cm}$ and the center of the screen was at the same level as the participants' eyes. The experimental set-up was identical to the fMRI study with the following three exceptions. The fixation cross was presented for $2,000 \mathrm{~ms}$ instead of $600 \mathrm{~ms}$ to ensure that the participants were really fixating on the same position before each trial start. The stimuli (prime as well as prime together with the target words) were presented on a gray background to minimize differences in luminance. Finally, the stimuli were presented 130 pixels below the middle of the screen to account for the fact that participants tend to fixate the center of the screen independent of the task (Bindemann, 2010). The participants also received four practice trials before starting the experiment to ensure that they understood all task instructions. They were newly calibrated before every experimental block and the average calibration error across all runs was $M=0.40^{\circ}, S D=0.20^{\circ}$. We excluded one subject from the analysis of the eye tracking data due to too low-data quality (the eyetracker did not manage to track this participant's pupil in a stable manner). Hence, 25 subjects were entered into the final analysis.

We first checked whether our independent sample from the eye tracking study reproduced similar behavioral results as the sample from the fMRI results. Therefore, we set up a GLMM with the AHA! experience as dependent variable of interest and the predictors accuracy, solution time, amount of search and perceived effort to find the solution as fixed effects. All subjects and CRA items were modeled as random intercept effects.

Subsequently, we set up a second GLMM using (a) the semantic distance to the solution word, (b1) frequency ([b2] the amount of compounds) and (c) word length of the most highly fixated word within the first $3 \mathrm{~s}$ after trial start and (d) un/related prime as predictors. The semantic distance of the most highly attended target word to the solution was estimated via cosine distance in exactly the same way as the un/related prime had been estimated (see further above). Word length served as covariate of no interest to control for the fact that longer words may be fixated more. Frequency was derived using the Google Ngram Viewer (https://books.google.com/ngrams) based on the year 2008. The amount of compounds represents an estimate of the amount of possible compounds that can be built with the respective word. This variable was derived by counting the total number of suggested meaningful compounds per word using the online platform http:// www.dict.cc (these lexical parameters have already been used in Becker et al., 2018 but without eye tracking). The lexical properties of frequency and amount of compounds of the most highly attended word after trial start are not independent but correlated $(r=.533)$. Thus due to colinearity, we decided to estimate both frequency and amount of compounds in a separate GLMM.

\section{3 | RESULTS}

\section{1 | Behavioral results}

The odds of having a solution accompanied with an AHA! experience increased with the factor of $1.78(p<.01)$ when the CRA problem was
TAB LE 2 Generalized linear mixed model [GLMM] resultsinfluence of solution time, amount of search for the solution, search effort, accuracy, and preceding semantically un/related prime to solution on the likelihood to report a solution with an AHA! experience

\begin{tabular}{llll} 
& \multicolumn{4}{l}{ AHA! experience } \\
\cline { 2 - 4 } Predictors & Odds ratios & \multicolumn{1}{l}{$\mathrm{Cl}$} & \multicolumn{1}{l}{$\boldsymbol{p}$} \\
\hline (intercept) & 14.26 & $6.03-33.71$ & $<.001$ \\
\hline Accuracy & 1.78 & $1.19-2.67$ & .005 \\
\hline Search & 0.85 & $0.72-1.00$ & .047 \\
\hline Search effort & 0.54 & $0.46-0.64$ & $<.001$ \\
\hline Prime: semantically related & 1.37 & $1.04-1.80$ & .025 \\
\hline Solution time & 0.96 & $0.94-0.97$ & $<.001$ \\
\hline Random effects & & & \\
\hline$\sigma^{2}$ & 3.29 & & \\
\hline ICC items & 0.01 & & \\
\hline ICC & & \\
\hline subjects & 0.41 & & \\
\hline Marginal $R^{2} /$ conditional $R^{2}$ & $0.279 / 0.579$ & & \\
\hline
\end{tabular}

Note. $\mathrm{Cl}$, confidence interval; $p, p$-value; ICC, intraclass correlation coefficient.

solved correctly. Concretely, accuracy was $90.88 \%$ (SD = 28.79\%) when the problem was solved with an AHA! experience, whereas accuracy dropped to $73.53 \%$ ( $S D=44.15 \%$ ) when the problem solution was accompanied without AHA! experience.

The odds of an AHA! experience increased with the factor of 1.37 $(p<.05)$ when a solution related compared to an unrelated prime was shown preceding the CRA problem presentation (see, Table 2).

On average, participants needed $14.26 \mathrm{~s}$ (SD = $10.61 \mathrm{~s}$ ) to solve a CRA problem accompanied with an AHA! experience, whereas they solved a CRA problem in $23.53 \mathrm{~s}(S D=14.58 \mathrm{~s})$ on average without AHA! experience. This difference is significant, the odds of having an AHA! experience decreases with the factor of $0.96(p<.001)$ with every second that the problem is not solved (see, Table 2, Figure 3).

Participants reported 0.93 possible but wrong solutions (search) on average $(S D=1.15)$ when they found the solution accompanied with an $\mathrm{AHA}$ ! experience. In case of a solution without AHA! experience, they reported 1.58 possible but wrong solutions on average $(S D=1.75)$. Note, it is possible that the search button press (2) could have a detrimental effect on the AHA! experience (see verbal overshadowing effect on insight solutions probably due to attention, Schooler \& Melcher, 1995). However, the proportion of CRA solutions accompanied with an AHA! in our study ( $M=58 \%$; $S D=21 \%$ ) was comparable to CRA solutions with an AHA! in another MR study using CRAs without search button press ( $M=56 \%, S D=18 \%$ ) (see, Jung-Beeman et al., 2004, p. 501).

The odds of having an AHA! experience decreases with the factor of $0.85(p<.05)$ with every new possible but wrong solution they report, even when controlling for solution time.

Finally, participants reported an effort to find the solution of 2.74 $(S D=1.39)$ when having found the solution accompanied with an AHA! experience and a search effort of $3.50(S D=1.10)$ when they did not 


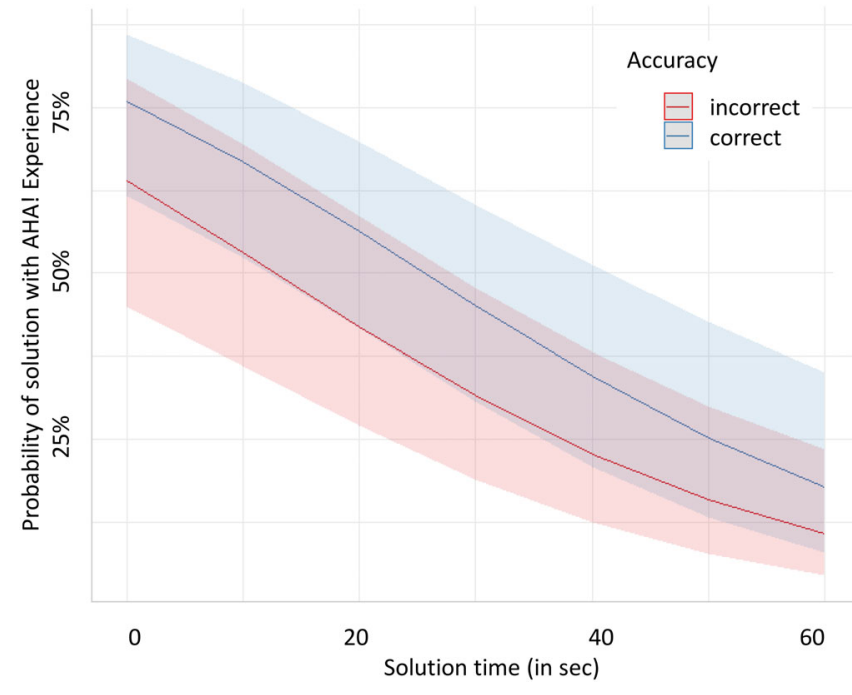

FIGURE 3 Predicted probability of solution accompanied with AHA! experience as function of solution time for correctly and incorrectly solved trials. Note. The regression lines represent the fitted values of solution time for correctly and incorrectly solved trials from the general linear mixed model in Table 2. The shaded areas around the regression lines represent the $95 \%$ confidence interval

find the solution accompanied with an AHA! experience on average. This difference in search effort was also significant: the odds of having an AHA! experience decreased with the factor of $0.54(p<.001)$ with every increasing unit of self-reported search effort (see, Table 2).

\section{2 | Functional imaging results}

\subsection{1 | Main effects of AHA and prime within first $3 \mathrm{~s}$ after trial start}

First, we investigated differences in brain activation during the first $3 \mathrm{~s}$ after trial start. Our hypothesis was that problems that are later solved with an AHA! experience are already processed differently shortly after trial start compared to problems that are later solved without AHA!. The random effects analysis of the main effect AHA versus noAHA (Related_Prime_AHA + Unrelated_Prime_AHA > Related_Prime_noAHA + Unrelated_Prime_noAHA) revealed a significant activation in the left insula [peak voxel $x ; y ; z(\mathrm{MNI})=-30,16,-2, t=4.33$; cluster size $=185$ voxels; $p=.001$ ] and in the right insula extending into inferior frontal gyrus [peak voxel $x ; y ; z(\mathrm{MNI})=32,14,-6, t=4.64$; cluster size $=206$ voxels; $p<.001]$ (see, Figure 4, Panel a). Note, the $p$-value in the square brackets always refers to the cluster-level FWE corrected $p$-value. The inverted contrast noAHA versus AHA (Related_Prime_noAHA + Unrelated_Prime_ noAHA > Related_Prime_AHA + Unrelated_Prime_AHA) revealed no significant clusters (the most important results including the respective cluster size are presented in Table 3).

Second, to assess whether the semantic relatedness of the prime to the solution influences the BOLD signal during trial start, we calculated two contrasts Related Prime versus Unrelated Prime (i.e., Related_Prime_AHA + Related_Prime_noAHA > Unrelated_Prime_AHA +
Unrelated_Prime_noAHA) and the reverse contrast. The first contrast did not reveal any significant clusters, neither did the reverse contrast. A visual inspection of the beta estimates of the four conditions at trial start (Related_Prime_AHA, Unrelated_Prime_AHA, Related_Prime_noAHA, Unrelated_Prime_noAHA) suggested no influence of the prime onto the observed difference in BOLD contrast between the AHA and noAHA condition (see, Figure 4, Panel c [bars 1 till 4]).

\subsubsection{Main effects of AHA and prime during search}

We investigated possible differences in brain activation during search for the solution (button press 2 ) between CRA items that were later solved with or without AHA! experience. The random effects analysis for the contrast noAHA > AHA during the search phase revealed no significant clusters at the voxel threshold $p<.001$. However, lowering this voxel-based threshold to $p<.005$ for exploratory purposes, revealed two significant clusters in the left superior temporal gyrus extending to the left insula [peak voxel $x ; y ; z(\mathrm{MNI})=-44,0,-16$; $t=3.89$, cluster size $=380$ voxels; $p=.001]$ as well as in the right superior temporal gyrus extending to right insula [peak voxel $x ; y ; z$ $(\mathrm{MNI})=50,-6,-8 ; t=4.43$, cluster size $=372$ voxels; $p=.001]$. The reverse contrast $\mathrm{AHA}>$ noAHA (with both voxel-based thresholds) revealed no significant clusters.

\subsection{3 | Main effects of AHA and prime during solution}

We investigated differences in brain activation during problem solution (button press 1). We hypothesized that more cognitive control areas should be activated in solutions accompanied without than with an AHA! experience during solution. The random effects analysis of the contrast noAHA > AHA (i.e., Related_Prime_noAHA + Unrelated_Prime_noAHA > Related_Prime_AHA + Unrelated_Prime_AHA) revealed a significant activation in the left anterior insula [peak voxel $x ; y ; z(\mathrm{MNI})=-30,26,-2$, $t=5.38$; cluster size $=356$ voxels, $p<.001]$, in the right anterior insula extending into the right inferior frontal gyurs [peak voxel $x ; y ; z(M N I)=32,20$, $-2, t=6.15$; cluster size $=716$ voxels, $p<.001]$, in bilateral superior medial frontal gyrus extending into the anterior and middle cingulum as well as to bilateral supplementary motor cortex [peak voxel $x ; y ; z(M N I)=-6,20,40$; $t=6.32$; cluster size $=3,159$ voxels, $p<.001]$, in left and right caudate nucleus [peak voxel $x ; y ; z(M N I)=-12,10,12 ; t=5.34$; cluster size $=729$ voxels, $p<.001$ ], in a cluster located in the thalamus extending to the brain stem [peak voxel $x ; y ; z(M N I) 8,-10,-2 ; t=5.09$; cluster size $=272$ voxels, $p<.001]$ and in right inferior frontal gyrus, pars opercularis $[x ; y ; z(M N I)$ 58,20,12; $t=4.51$; cluster size $=198$ voxels, $p<.001$ ] (see, Figure 4, Panel b, Panel c [bars 5 till 8]).

The reverse contrast AHA > noAHA (i.e., Related_Prime_AHA + Unrelated-Prime_AHA > Related_Prime_noAHA + Unrelated_Prime_ noAHA) revealed a significant cluster in the right rolandic operculum extending into white matter [peak voxel $x ; y ; z(M N I)=48,-14,22$; $t=3.66$; cluster size $=195$ voxels, $p<.001]$. As hypothesized, a cluster in the left hippocampus could also be observed but it did not meet the cluster-based cutoff criterion [peak voxel $x ; y ; z(M N I)=4-24,-6, .22$; 
(a) trial start: AHA > noAHA

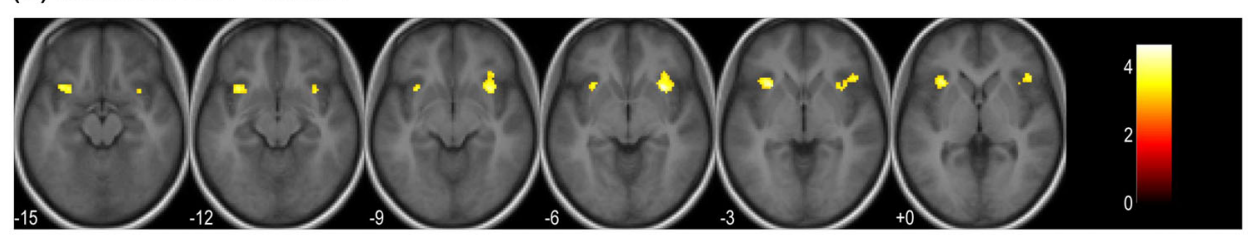

(b) solution: noAHA > AHA

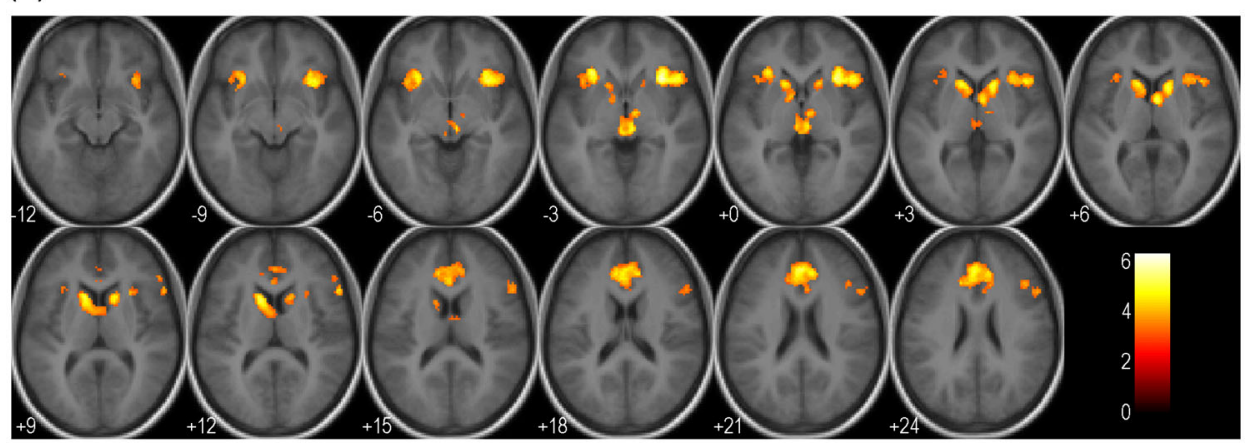

(c) beta values from peak voxel in left anterior insula for all modelled events

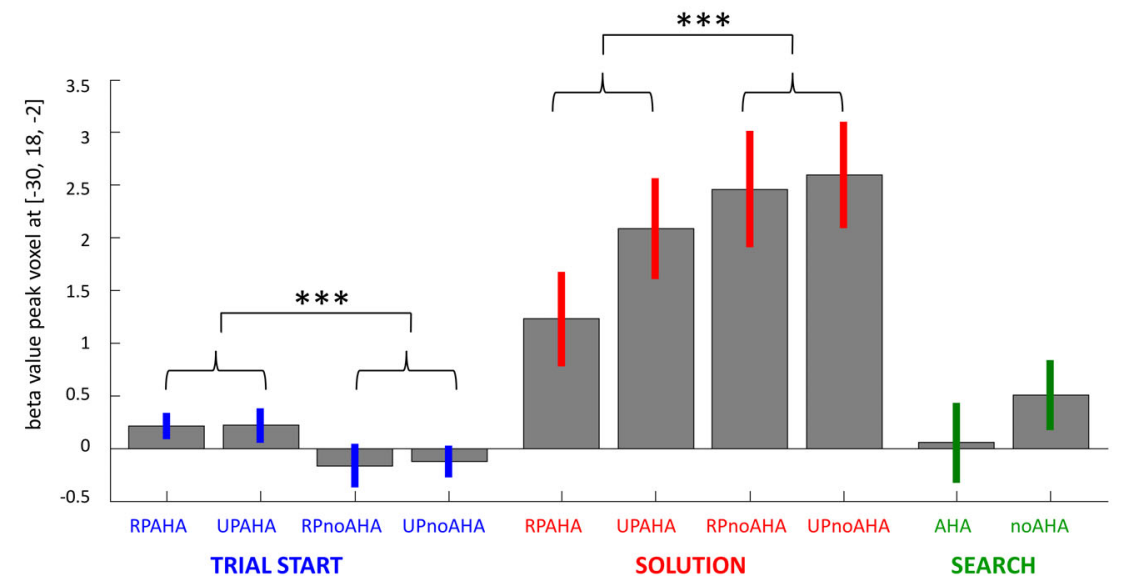

FIGURE 4 fMRI results from the full factorial design-brain activation during trial start (AHA > noAHA) and solution (noAHA > AHA) as well as beta values from peak voxel in left anterior insula for all modeled events. Note. Panel (a) brain activation within the first $3 \mathrm{~s}$ after trial onset related to the contrast: Related_Prime_AHA + Unrelated_Prime_AHA > Related_Prime_noAHA + Unrelated_Prime_noAHA; clusters are wholebrain FWE corrected for multiple comparisons at $p<.01$; Panel (b) brain activation during solution related to the contrast: Related_Prime_noAHA + Unrelated_Prime_noAHA > Related_Prime_AHA + Unrelated_Prime_AHA; clusters are whole-brain FWE corrected for multiple comparisons at $p<.01$; Panel (c) Beta values for peak voxel in left anterior insula [-30 18 2] from the conjunction analysis for all estimated events in full factorial design. The bars with the blue error bars correspond to four different conditions within the first $3 \mathrm{~s}$ after trial start; the bars with the red error bars correspond to four conditions during solution and the two bars with the green error bars correspond to the search phase. The conditions are the following: RPAHA = Related_Prime_AHA, UPAHA = Unrelated_Prime_AHA, RPnoAHA = Related_Prime_noAHA,

UPnoAHA = Unrelated_Prime_noAHA (see, Table 1); error bars represent $90 \%$ confidence intervals; ***represents a significant difference (voxelbased threshold $p<.001$; cluster-based FWE corrected threshold $p<.01$ ) between beta weights in the cluster of left anterior insula including the peak voxel $[-30,18,-2]$ of respective events

$t=4.37$; cluster size $=80$ voxels, $p=.056]$. However, when performing a ROI-based analysis with a broad mask including bilateral hippocampi, amygdalae, and parahippocampal gyri for the same contrast, activation in this region differed significantly $(t=1.95, p=.026)$.

To investigate the influence of the prime in this solution stage, we calculated two contrasts Related Prime $>$ Unrelated Prime (i.e., Related_Prime_AHA + Related_Prime_noAHA > Unrelated_Prime_ $\mathrm{AHA}+$ Unrelated_Prime_noAHA) and the reverse contrast for the solution. Both contrasts did not reveal any significant clusters. However, when specifically investigating the influence of the semantically unrelated prime for solutions accompanied with an AHA! experience (Unrelated_Prime_AHA > Related_Prime_AHA) at the solution stage, we observed a significant cluster in left anterior insula extending into left inferior frontal gyrus [peak voxel $x ; y ; z(M N I)=-28,22,4 ; t=4.94$; cluster size: 811 voxels; $p<.001$ ], the right anterior insula spreading to right inferior frontal gyrus, pars orbitalis [peak voxel $x ; y ; z(M N I)=46$, $38,-14 ; t=4.66$, cluster size: 557 voxels; $p<.001$ ], right inferior frontal gyrus, pars opercularis [peak voxel $x ; y ; z(M N I)=52,20,32 ; t=4.60$; 
TAB LE 3 MNI coordinates and T-scores of hemodynamically activated brain areas from different contrasts of the second level full factorial model

\begin{tabular}{|c|c|c|c|c|c|c|c|}
\hline & Side & \multicolumn{3}{|c|}{ Peak voxel MNI coordinates (mm) } & $\frac{\text { Cluster size }}{\text { (voxel) }}$ & Peak $t$ score & $\frac{p \text {-value }}{\text { (FWE) }}$ \\
\hline \multicolumn{8}{|c|}{ fMRI results during solution phase-trial start: $A H A>$ noAHA solutions } \\
\hline Anterior insula & L & -30 & 16 & -2 & 185 & 4.33 & .001 \\
\hline \multicolumn{8}{|c|}{ fMRI results during solution phase-solution: $A H A$ > noAHA solutions } \\
\hline Hippocampus** & L & -24 & -6 & -22 & 80 & 4.37 & .056 \\
\hline \multicolumn{8}{|c|}{ fMRI results during solution phase-solution: noAHA > AHA solutions } \\
\hline $\begin{array}{l}\text { Medial superior frontal gyrus extending to ACC, } \\
\text { middle cingulum and SMA }\end{array}$ & L/R & -6 & 20 & 40 & 3,159 & 6.32 & .000 \\
\hline $\begin{array}{l}\text { Anterior insula extending to right inferior } \\
\text { frontal gyurs, pars triangularis }\end{array}$ & $\mathrm{R}$ & 32 & 20 & -2 & 716 & 6,15 & .000 \\
\hline Thalamus extending to brainstem & L & 8 & -10 & -2 & 272 & 5.09 & .000 \\
\hline Inferior frontal gyrus, pars opercularis & $\mathrm{R}$ & 58 & 20 & 12 & 198 & 4.51 & .000 \\
\hline \multicolumn{8}{|c|}{ fMRI results for conjunction analysis: (trial start: $A H A$ > noAHA) and (solution: noAHA > AHA) } \\
\hline $\begin{array}{l}\text { Anterior insula extending to inferior } \\
\text { frontal Gyrus, pars triangularis }\end{array}$ & $\mathrm{R}$ & 46 & 22 & 0 & 180 & 3.79 & .001 \\
\hline Anterior insula & L & -30 & 18 & -2 & 87 & 4.16 & .046 \\
\hline
\end{tabular}

Note. Clusters are whole-brain FWE corrected for multiple comparisons at $p<.01$ (for conjunction analysis-exploratory): $p<.05$ ); L, left; R, right; size (voxel), cluster size, peak $t$ score, maximimum $t$ value of significantly activated clusters. Trial start-means mean value of first $3 \mathrm{~s}$ after trial start; ACC, anterior cingulate cortex, SMA, supplementary motor cortex, for region marked with asterisk: did not reach significance $(p=.056)$ in whole brain analysis.

cluster size: 158 voxels; $p=.002$ ], bilateral superior medial prefrontal gyrus extending into left anterior cingulum [peak voxel $x ; y ; z(M N I)=-2$, 32, 38; $t=4.54$; cluster size: 607 voxels; $p<.001]$. The reverse contrast (Related_Prime_AHA > Unrelated_Prime_AHA) did not reveal any significant results. Furthermore, there was no effect of the prime on solutions solved without an AHA! experience (Related_Prime_noAHA > Unrelated_ Prime_noAHA; Unrelated_Prime_noAHA > Related_Prime_noAHA).

Visual inspection of the beta estimates of the four conditions during the solution (Related_Prime_AHA, Unrelated_Prime_AHA, Related_Prime_noAHA, Unrelated_Prime_noAHA) confirmed the influence of the prime for CRA items solved with an AHA! experience (see, Figure 4, Panel c [bars 5 till 8]).

\subsection{4 | Conjunction and interaction analysis for solutions with and without AHA during trial start and solution}

Finally to investigate whether processes underlying solutions experiences with and without AHA activate similar brain areas but at different time points (AHA during trial start, noAHA during solution), we first conducted a conjunction analysis to isolate the shared significant activation during both solution phases: The contrast (trial start: $\mathrm{AHA}>$ noAHA) and (solution: noAHA > AHA) revealed two significant clusters in the left insula [peak voxel $x ; y ; z(M N I)=-30,18,-2 ; t=4.16$; cluster size: 87 voxels; $p=.046$ ] and right insula extending into right inferior frontal gyrus [peak voxel $x ; y ; z(\mathrm{MNI})=46,22,0 ; t=3.79$; cluster size: 180 voxels; $p=.001]$ ).

Second, we conducted a whole-brain interaction analysis masked with the significant clusters from the conjunction analysis with the contrast (trial start: AHA - noAHA) - (solution: AHA - noAHA). The interaction analysis revealed a significant solution experience $\times$ solution phase interaction in both insula clusters that were already significant in the conjunction analysis (left insula: [peak voxel $x ; y ; z(\mathrm{MNI})=-30$, $20,-2 ; t=6.13$; cluster size: 87 voxels; $p=.040]$; right insula extending into the right inferior frontal gyrus [peak voxel $x ; y ; z(M N I)=32,22,-4$; $t=7.07$; cluster size: 180 voxels; $p=.001]$ ).

\subsubsection{Conjunction analysis during solution}

Finally, to investigate whether the prime effect for solutions with $A H A$ ! in the BOLD response during solution is related to the differences in the BOLD contrast noAHA - AHA during solution, we additionally conducted a conjunction analysis of the contrasts noAHA > AHA \& Unrelated_Prime_AHA > Related_Prime_AHA both during solution. This analysis revealed three significant clusters in right [peak voxel $x ; y ; z(\mathrm{MNI})=34,24,-2 ; t=4.66$; cluster size: 287 voxels; $p<.001$ ] and left [peak voxel $x ; y ; z(\mathrm{MNI})=-28,20,-6$; $t=4.60$; cluster size: 288 voxels; $p<.001]$ anterior insulae and in the 
left superior medial frontal gyrus extending into the ACC and supplementary motor area [peak voxel $x ; y ; z(\mathrm{MNI})=-2,32,38 ; t=4.54$; cluster size: 471 voxels; $p<.001$.

\section{3 | Eye tracking results}

The behavioral data from the eye tracking sample produced comparable results to the data of the $\mathrm{fMRI}$ sample. Accuracy, reaction time and search effort served as significant predictors for a solution accompanied by an AHA! experience. The odds of having an AHA! increased with the factor of $2.99(p<.001)$ when the solution was correct. The odds of having an AHA! decreased to the factor of $0.96(p<.001)$ with increasing solution time and it also decreased to the factor of 0.63 $(p<.001)$ with increasing search effort. In this GLMM, the amount of search did not reach significance although participants reported to have found 0.45 possible but wrong solutions less when solving the CRA with AHA! and this difference is significant $t(850.6)=7.670$, $p<.001$ ). However, this difference in search vanishes when accounting for the increased solution time for problems solved without AHA!.

In a second GLMM modeling the influence of the lexical properties of the most highly attended word in the first $3 \mathrm{~s}$ after trial start as well as the un/related prime, frequency, and the un/related prime served as significant predictors. Specifically, the odds of having a solution with AHA! decrease with increasing frequency of the most highly attended word after trial start (factor of $0.82, p=.005$ ). The semantic distance of the most highly attended word after trial start does not predict a solution accompanied by an AHA!. However, the odds of having an AHA! increase to the factor of $1.74(p<.001)$ when a semantically related prime is presented in general, that is, independent of the focus of attention within the first $3 \mathrm{~s}$ after trial start (see, Table 4).

TAB LE 4 GLMM results-influence of frequency, word length, and semantic distance of most highly attended word within the first $3 \mathrm{~s}$ after trial start as well as un/related prime on the likelihood to report a solution with an AHA! experience

\begin{tabular}{|c|c|c|c|}
\hline \multirow[b]{2}{*}{ Predictors } & \multicolumn{3}{|c|}{ AHA! experience } \\
\hline & Odds ratios & $\mathrm{Cl}$ & $p$ \\
\hline (intercept) & 1.52 & $1.07-2.14$ & .018 \\
\hline Frequency (first $3 \mathrm{~s}$ ) & 0.82 & $0.72-0.94$ & .005 \\
\hline Word length (first $3 \mathrm{~s}$ ) & 0.94 & $0.82-1.08$ & .379 \\
\hline Semantic distance (first $3 \mathrm{~s}$ ) & 0.97 & $0.85-1.11$ & .654 \\
\hline Prime: semantically related & 1.74 & $1.36-2.22$ & $<.001$ \\
\hline \multicolumn{4}{|l|}{ Random effects } \\
\hline$\sigma^{2}$ & 3.29 & & \\
\hline$I C C_{\text {items }}$ & 0.03 & & \\
\hline $\mathrm{ICC}_{\text {subjects }}$ & 0.13 & & \\
\hline Observations & 1,360 & & \\
\hline Marginal $R^{2} /$ conditional $R^{2}$ & $0.028 / 0.188$ & & \\
\hline
\end{tabular}

Note. $\mathrm{Cl}$, confidence interval; ICC, intraclass correlation coefficient. The most highly attended word per trial was estimated via the highest amount of fixations within the first $3 \mathrm{~s}$ after trial start.
Finally, in a third GLMM when exchanging the nonindependent predictors frequency with the amount of compounds, the latter variable also significantly predicts the AHA! experience (word length, semantic distance and un/related prime do not change significantly). The odds for a solution accompanied by an AHA! decrease (factor of 0.86 , $p=.032$ ) the higher the amount of compounds of the most highly attended word in the first $3 \mathrm{~s}$ after trial start is.

\section{4 | DISCUSSION}

In the present study, we investigated the question how solutions accompanied with and without an AHA! experience differ by not only focusing on the time point of the solution, but also by taking different phases of the solution process into account. As earlier priming studies suggested, we hypothesized that solutions accompanied by an AHA! experience are not spontaneously generated during solution but already relate to prior subliminal processing (Kounios \& Beeman, 2014). In line with results from Aziz-Zadeh et al. (2009), we hypothesized that solution-relevant processing takes place earlier (shortly after trial start) and therefore retrieving the solution word should require less conscious effort for problems solved with than without AHA! experience. Our results support this hypothesis: We could show that problems solved with an AHA! experience are not only solved faster and are more accurate but they already exhibit more bilateral anterior insula activation within the first $3 \mathrm{~s}$ after trial start compared to problems solved without an AHA! experience (note, this replicates the results by Aziz-Zadeh et al. (2009) despite our lower sampling rate). Although the likelihood for an AHA! experience increased when a semantically related prime in contrast to an unrelated prime was presented, we failed to find any evidence that this increased insular activity within the first $3 \mathrm{~s}$ after trial start was a function of semantic priming. This finding suggests that the observed differential activation after trial start is not due to solution-relevant associations caused by the semantically related prime. We did not expect that given that we replicated the behavioral priming effect (increased likelihood for an AHA! experience due to the presentation of a semantically related prime) from earlier studies (Bowden, 1997; Bowden \& Jung-Beeman, 2003b). It is possible that the continuous presentation of the prime attenuated the (early) BOLD response so that no difference could be detected as a function of the prime. However, this seems only little plausible given that we do observe a late priming related difference in $B O L D$ response in prefrontal areas during the solution phase but only for solutions accompanied by an AHA!. Moreover, a conjunction analysis revealed that the salience network (bilateral insula and ACC) is commonly activated for the contrast noAHA $>$ AHA and Unrelated_Prime_AHA $>$ Related_Prime_AHA during solution. This suggests that the influence of the prime in solutions with AHA! may drive the effects of the BOLD contrast noAHA > AHA during solution. Therefore, we assume that the replicated behavioral priming effect on the AHA! experience is more likely related to a late influence of the prime during the solution phase rather than after trial onset. 
In line with a more effortful solution process for problems solved without AHA! experience, we observed more search attempts and self-reported search effort for this solution experience.

In addition, although we did not observe any differential brain activity at a voxel-based threshold of $p<.001$ during search, when lowering the voxel-based threshold to $p<.005$ for exploratory purposes, we observed increased bilateral superior temporal gyrus activity for problems later solved without than with an AHA! experience. The superior temporal gyrus (STG) has been associated with semantic processes and semantic integration (Friederici, Rüschemeyer, Hahne, \& Fiebach, 2003; Mazoyer et al., 1993; Visser \& Lambon Ralph, 2011). In context of insight problem solving, especially the right STG is assumed to integrate information across distant semantic relations (Jung-Beeman et al., 2004). Hence, the fMRI results during the search phase point at least in the same direction as the behavioral data in the sense that participants may need to search more thoroughly in the semantic domain for a potential solution for problems later solved without than with AHA! solutions. This would be in line with the hypothesis that solution-relevant processing takes place later and the whole solution process is therefore perceived as more effortful for problems later solved without than with AHA! experience.

Further in line with a more effortful solution process is the increased prefrontal activity including anterior insular, right inferior, right middle and superior medial frontal gyrus as well as caudate nuclei activity for problems solved without than with an AHA! experience during the solution phase. The caudate nuclei have been shown to be sensitive to changes in meaning of words and are therefore assumed to play a role in monitoring and controlling language in use (Crinion et al., 2006). Furthermore the caudate as part of the dorsal striatum has also been associated with cognitive control and effort (MacDonald et al., 2014; Mestres-Missé, Turner, \& Friederici, 2012; Robertson, Hiebert, Seergobin, Owen, \& MacDonald, 2015). Similarly, the right inferior and medial frontal cortex are also implicated in cognitive control (Aron, Robbins, \& Poldrack, 2004; Duncan \& Owen, 2000; MacDonald, Cohen, Stenger, \& Carter, 2000; Ridderinkhof, Ullsperger, Crone, \& Nieuwenhuis, 2004; Robertson et al., 2015). In particular, the posterior medial frontal cortex has been associated specifically with performance monitoring like the detection of response conflict or decision uncertainty and the capacity to signal the need for performance adjustment (for a meta-analysis, see Ridderinkhof et al., 2004). All in all, this suggests that solutions accompanied without AHA! experience require a higher employment of brain areas associated with cognitive control during solution possibly in order to retrieve the solution word from memory. However, we cannot entirely exclude that some of the brain signals measured during the final solution stage also refers to postsolution verification processes. Especially, the medial frontal gyrus activity may suggest that the subjects were not certain about their final solution and therefore may have encountered a response conflict. This would be in line with the fact that solutions accompanied without AHA! were more often incorrect. Moreover, the supplementary motor area (as part of the medial frontal cortex) plays a role in initiating and executing speech (Price, 2010). This suggests that participants were more strongly engaged in speech production processes during solutions accompanied without AHA! experience. Hence, it could be that participants were more strongly verbalizing the solution (accompanied without AHA!) silently in combination with the target words to verify its correctness.

Although the observed cluster in the left hippocampal gyrus did not reach significance in the whole-brain analysis, the ROI-based analysis suggests that solutions accompanied with AHA! experience exhibit increased hippocampal activity as had been shown before for insight problems during solution (Jung-Beeman et al., 2004; Kizilirmak et al., 2016; Luo \& Niki, 2003; Zhao et al., 2013). This hippocampal activation has been associated with detecting novel associations in a problem solving context (Kizilirmak et al., 2016; Luo \& Niki, 2003) but it is widely recognized for its role in memory retrieval in general (e.g., Carr, Jadhav, \& Frank, 2011; Ranganath, Cohen, Dam, \& D'Esposito, 2004). Therefore, we assume stronger hippocampal activation for solutions accompanied with than without an AHA! to be the result of sudden retrieval of solution relevant associations leading to the sudden awareness of the final solution.

In addition, we found increased activation in right rolandic operculum during solution for solutions accompanied with than without AHA! experience. To our knowledge, this has not been reported in the context of insight problem solving before. Although the rolandic operculum has been associated with language production and articulatory impairments (Brown, Ingham, Ingham, Laird, \& Fox, 2005; Tonkonogy \& Goodglass, 1981), it has also been associated with the tip-of-the-tongue phenomenon (TOT) and phonological retrieval (Shafto, Burke, Stamatakis, Tam, \& Tyler, 2007). TOT describes frustrating word-finding failures where people are temporarily unable to produce a word that they know. It is assumed that TOTs happen when semantic information of the respective word has already been accessed producing a strong feeling of knowing but sufficient phonological information cannot be retrieved at this particular moment (Burke, MacKay, Worthley, \& Wade, 1991; Cross \& Burke, 2004). Although speculative but solution-relevant semantic information in problems solved with AHA! could already be retrieved before the solution. Therefore, the increased rolandic operculum activity could possibly reflect the retrieval of the still outstanding phonological information of the solution word.

Further in line with our hypothesis that both solution experiences differ in terms of time-related solution-relevant processing, is the fact that we observed a significant solution experience $\times$ solution phase interaction. That is to say, we observed increased bilateral insular activity after trial start for problems solved with compared to without AHA! experience while the opposite pattern was the case during solution. Although we did not hypothesize this interaction beforehand, this finding further suggests that the anterior insula plays a key role in solution-relevant processing of verbal insight problems like CRAs but the timing and extent of activation differs between both solution experiences. That is to say, for problems later solved with AHA!, possible relevant processing in the anterior insula seems to take place already shortly after trial start, while for problems later solved without $\mathrm{AHA}$ ! this may take place only later during solution but in combination with conscious effort. 
What these fMRI results, however, leave unanswered is why problems later solved with an AHA! experience are already differentially processed in the anterior insula shortly after trial start. This is especially the case, since we did not find evidence for an influence of the prime onto early processing in bilateral anterior insula as we would have expected.

Kounios and colleagues report task-unrelated mental preparation facilitating later solutions accompanied by an AHA! experience that is associated with heightened activity in medial frontal and temporal areas before problem presentation (Kounios et al., 2006). However, these reported distinct brain states cannot be task-related as participants have not been exposed to the respective CRA item, yet. In contrast, we believe that the early difference in insular activation reported here is more likely task-related because we find a similar activation during solution of the problem (but without AHA!).

Aziz-Zadeh et al. (2009) attributed the early increased bilateral anterior insula activation for problems solved with AHA! to its general language function. Indeed, this brain region is often coactivated with the inferior frontal gyrus in general language related tasks (for a metaanalysis, see Ardila et al., 2014).

Although the insula has also been considered a limbic region due to its implication in affective and regulatory functions like emotional responses and empathy (Flynn, Benson, \& Ardila, 1999; Singer, Critchley, \& Preuschoff, 2009), recent evidence from network analyses suggests a critical role especially of the anterior part of the insula in highlevel cognitive control and attentional processes (Chang, Yarkoni, Khaw, \& Sanfey, 2012; Menon \& Uddin, 2010). According to a functional parcellation study, the dorsal part of the anterior insula is functionally involved in cognitive processes such as task switching, inhibition, error processing, and conflict (Chang et al. 2012; Uddin, 2015). Congruently, the insula has been reported to be involved in verbal creative tasks requiring inhibition of dominant but task-irrelevant associations during solution (Tang et al., 2015; Wu, Knoblich, \& Luo, 2013).

However, we argue that the insula's role especially as part of the "salience network" is a promising explanation for the observed brain differences in solution experience shortly after trial start (Uddin, 2015). It has been suggested that the anterior insula in combination with the ACC form a "salience network" that functions to detect salient events and subsequently switches between other large-scale networks involved in externally oriented attention and internally oriented cognition for optimized access to attention and working memory resources (Menon \& Uddin, 2010; Sridharan, Levitin, \& Menon, 2008). Hence, the salience network and the anterior insulae as its integral hub is assumed to segregate the most relevant internal and external stimuli to guide behavior (Menon \& Uddin, 2010).

This assumed role of the anterior insula could explain the observed time-delayed differences in this region between both solution experiences. For problems later solved with an AHA!, solution-relevant words could be detected early and subsequently be processed preferentially (with more attributed attentional resources) leading to a more efficient (faster and less effortful) solution process. If this was true then one would expect early preferentially processed (that is most highly attended) words to be solution-relevant. The eye tracking results from the control experiment support this hypothesis. By investigating the lexical properties of the most highly attended word during early problem solving (first $3 \mathrm{~s}$ after trial start), we found that frequency and the amount of possible compounds negatively predict the likelihood for a later solution with AHA! experience. Specifically, the smaller the frequency and the amount of possible compounds of the most highly attended target the more likely was an AHA! experience (note, both predictors are not independent of each other). Fixating those words that are less common and that have less possible compounds is solution-relevant and can make the solution process more efficient because this may reduce the amount of possible solutions to search for. Given that we did not find fMRI evidence for the semantic prime to be related to the solution experience after trial start (nor between the semantic relationship of the most highly attended word and the solution), frequency and the amount of compounds of the attended word might explain the early insular activation for solutions with an AHA! experience.

However, it is important to note that the insular activity in the context of the salience network more likely represents general solutionrelevant processing (e.g., directing attentional resources to the most relevant external or internal stimuli) and no specific type of language related processing (e.g., only activation of semantically related associations) (Mennon \& Uddin, 2010). Salvi, Bricolo, Franconeri, Kounios, and Beeman (2015) also studied eye movements during CRA problem solving. They did not combine this with fMRI but they report dynamic differences in attention between both solution experiences before the trial starts and during problem solution (Salvi et al., 2015).

Finally, the present study provides evidence that solutions with and without AHA! differ in timing when solution-relevant processing takes place. We report fMRI results indicating early processing in the anterior insula for problems whose solutions are later accompanied with an AHA! experience. Surprisingly, no evidence for semantic priming could be found to account for this early difference in brain activation, as implied by the literature. Instead, first evidence points to the direction that early processing related to solutions with AHA! refers to reducing the amount of possible solutions to search for. The latter could lead to a more efficient solution process giving rise to the observed behavioral and fMRI differences between both solution experiences. However, only by combining $\mathrm{fMRI}$ and eye tracking methods the relationship between different lexical properties, brain activation, and allocation of overt attention can be directly analyzed. Therefore, future studies should ideally combine both methods when investigating the AHA! experience.

Nevertheless, this study demonstrates the importance of analyzing different phases of the solution process to get a better understanding of the (time-delayed) mechanism underlying both solution experiences.

\section{ACKNOWLEDGMENTS}

This work was supported by the German Science Foundation (SFB 936/C7; M.B.), the German Science Foundation (DFG KU 3322/1-1; S.K.), the European Research Council (ERC-2016-StG-Self-Control677804; S.K.). We want to thank Dr. Gregor Wiedemann for providing 
the semantic distances between the word pairs and Daniel Kutzner as well as Christoph Schäde for acquiring the fMRI data.

\section{CONFLICT OF INTEREST}

The authors declare no conflict of interest.

\section{ORCID}

Maxi Becker (1D) https://orcid.org/0000-0002-9430-4077

Tobias Sommer (D) https://orcid.org/0000-0002-3504-7357

Simone Kühn (D) https://orcid.org/0000-0001-6823-7969

\section{REFERENCES}

Ardila, A., Bernal, B., \& Rosselli, M. (2014). Participation of the insula in language revisited: a meta-analytic connectivity study. Journal of $\mathrm{Neu}$ rolinguistics, 29, 31-41.

Aron, A. R., Robbins, T. W., \& Poldrack, R. A. (2004). Inhibition and the right inferior frontal cortex. Trends in Cognitive Sciences, 8(4), 170-177.

Aziz-Zadeh, L., Kaplan, J. T., \& lacoboni, M. (2009). "Aha!": The neural correlates of verbal insight solutions. Human Brain Mapping, 30(3), 908-916.

Baayen, R. H., Davidson, D. J., \& Bates, D. M. (2008). Mixed-effects modeling with crossed random effects for subjects and items. Journal of Memory and Language, 59(4), 390-412.

Bates, D., Maechler, M., Bolker, B., \& Walker, S. (2014). Ime4: Linear mixed-effects models using Eigen and S4. R package version 1.0-6.

Becker, M., Sommer, T., Kühn, S. (in press). Inferior frontal gyrus involvement during search and solution in verbal creative problem solving: $\mathrm{A}$ parametric fMRI study.

Becker, M., Wiedemann, G., \& Kühn, S. (2018). Quantifying insightful problem solving: A modified compound remote associates paradigm using lexical priming to parametrically modulate different sources of task difficulty. Psychological Research. https://doi.org/10.1007/s00426018-1042-3

Bindemann, M. (2010). Scene and screen center bias early eye movements in scene viewing. Vision Research, 50(23), 2577-2587.

Bowden, E. M. (1997). The effect of reportable and unreportable hints on anagram solution and the aha! Experience. Consciousness and Cognition, 6(4), 545-573.

Bowden, E. M., \& Jung-Beeman, M. (2003a). Normative data for 144 compound remote associate problems. Behavior Research Methods, Instruments, \& Computers, 35(4), 634-639.

Bowden, E. M., \& Jung-Beeman, M. (2003b). Aha! Insight experience correlates with solution activation in the right hemisphere. Psychonomic Bulletin \& Review, 10(3), 730-737.

Bowden, E. M., \& Jung-Beeman, M. (2007). Methods for investigating the neural components of insight. Methods, 42(1), 87-99.

Bowden, E. M., Jung-Beeman, M., Fleck, J., \& Kounios, J. (2005). New approaches to demystifying insight. Trends in Cognitive Sciences, 9(7), 322-328.

Brainard, D. H. (1997). The psychophysics toolbox. Spatial Vision, 10, 433-436.

Brett M., Anton, J., Valabregue, R., Poline J.(2002). Region of interest analysis using an SPM toolbox [abstract]. Presented at the 8th International Conference on Functional Mapping of the Human Brain, June 2-6, Sendai, Japan. Available on CD-ROM in Neurolmage, Vol 16, No 2.

Brown, S., Ingham, R. J., Ingham, J. C., Laird, A. R., \& Fox, P. T. (2005). Stuttered and fluent speech production: An ALE meta-analysis of functional neuroimaging studies. Human Brain Mapping, 25(1), 105-117.
Burke, D. M., MacKay, D. G., Worthley, J. S., \& Wade, E. (1991). On the tip of the tongue: What causes word finding failures in young and older adults? Journal of Memory and Language, 30(5), 542-579.

Carr, M. F., Jadhav, S. P., \& Frank, L. M. (2011). Hippocampal replay in the awake state: A potential substrate for memory consolidation and retrieval. Nature Neuroscience, 14(2), 147-153.

Chang, L. J., Yarkoni, T., Khaw, M. W., \& Sanfey, A. G. (2012). Decoding the role of the insula in human cognition: Functional parcellation and large-scale reverse inference. Cerebral Cortex, 23(3), 739-749.

$R$ Core Team. (2014). $R$ : A language and environment for statistical computing (p. 2014). Vienna, Austria: R Foundation for Statistical Computing.

Crinion, J., Turner, R., Grogan, A., Hanakawa, T., Noppeney, U., Devlin, J. T., ... Usui, K. (2006). Language control in the bilingual brain. Science, 312(5779), 1537-1540.

Cross, E. S., \& Burke, D. M. (2004). Do alternative names block young and older adults' retrieval of proper names? Brain and Language, 89(1), 174-181.

Danek, A. H., Wiley, J., \& Öllinger, M. (2016). Solving classical insight problems without aha! experience: 9 dot, 8 coin, and matchstick arithmetic problems. The Journal of Problem Solving, 9(1), 4.

Duncan, J., \& Owen, A. M. (2000). Common regions of the human frontal lobe recruited by diverse cognitive demands. Trends in Neurosciences, 23(10), 475-483.

Eklund, A., Nichols, T. E., \& Knutsson, H. (2016). Cluster failure: Why fMRI inferences for spatial extent have inflated false-positive rates. Proceedings of the National Academy of Sciences, 113(28), 7900-7905.

Evans, A. C., Collins, D. L., Mills, S. R., Brown, E. D., Kelly, R. L., \& Peters, T. M. (1993. 3D statistical neuroanatomical models from $305 \mathrm{MRI}$ volumes. Nuclear Science Symposium and Medical Imaging Conference, 1993 IEEE Conference Record (pp. 1813-1817). IEEE.

Flynn, F. G., Benson, D. F., \& Ardila, A. (1999). Anatomy of the insula Functional and clinical correlates. Aphasiology, 13(1), 55-78.

Friederici, A. D., Rüschemeyer, S.-A., Hahne, A., \& Fiebach, C. J. (2003). The role of left inferior frontal gyrus and superior temporal cortex in sentence comprehension: Localizing syntactic and semantic processes. Cerebral Cortex, 13, 170-177.

Jung-Beeman, M., Bowden, E. M., Haberman, J., Frymiare, J. L., ArambelLiu, S., Greenblatt, R., ... Kounios, J. (2004). Neural activity when people solve verbal problems with insight. PLoS Biology, 2(4), 500-510.

Kizilirmak, J. M., Thuerich, H., Folta-Schoofs, K., Schott, B. H., \& Richardson-Klavehn, A. (2016). Neural correlates of learning from induced insight: A case for reward-based episodic encoding. Frontiers in Psychology, 7, 1693.

Knoblich, G., Ohlsson, S., Haider, H., \& Rhenius, D. (1999). Constraint relaxation and chunk decomposition in insight problem solving. Journal of Experimental Psychology: Learning, Memory, and Cognition, 25(6), 1534.

Kounios, J., \& Beeman, M. (2014). The cognitive neuroscience of insight. Annual Review of Psychology, 65, 71-93.

Kounios, J., Frymiare, J. L., Bowden, E. M., Fleck, J. I., Subramaniam, K., Parrish, T. B., ... Jung-Beeman, M. (2006). The prepared mind neural activity prior to problem presentation predicts subsequent solution by sudden insight. Psychological Science, 17(10), 882-890.

Lüdecke D (2018). ggeffects: Tidy Data Frames of Marginal Effects from Regression Models. Journal of Open Source Software, 3(26), 772. http://doi.org/10.21105/joss.00772

Ludmer, R., Dudai, Y., \& Rubin, N. (2011). Uncovering camouflage: Amygdala activation predicts long-term memory of induced perceptual insight. Neuron, 69, 1002-1014.

Luo, J., \& Niki, K. (2003). Function of hippocampus in "insight" of problem solving. Hippocampus, 13(3), 316-323.

Luo, J., Niki, K., \& Phillips, S. (2004). Neural correlates of the 'Aha! reaction'. Neuroreport, 15(13), 2013-2017.

MacDonald, A. A., Seergobin, K. N., Tamjeedi, R., Owen, A. M., Provost, J. S., Monchi, O., ... MacDonald, P. A. (2014). Examining dorsal 
striatum in cognitive effort using Parkinson's disease and fMRI. Annals of Clinical and Translational Neurology, 1(6), 390-400.

MacDonald, A. W., Cohen, J. D., Stenger, V. A., \& Carter, C. S. (2000). Dissociating the role of the dorsolateral prefrontal and anterior cingulated cortex in cognitive control. Science, 288(5472), 1835-1838.

Mazoyer, B. M., Tzourio, N., Frak, V., Syrota, A., Murayama, N., Levrier, O., ... Mehler, J. (1993). The cortical representation of speech. Journal of Cognitive Neuroscience, 5, 467-479.

Mednick, S. A. (1962). The associative basis of the creative process. Psychological Review, 69, 220-232.

Menon, V., \& Uddin, L. Q. (2010). Saliency, switching, attention and control: A network model of insula function. Brain Structure and Function, 214(5-6), 655-667.

Mestres-Missé, A., Turner, R., \& Friederici, A. D. (2012). An anteriorposterior gradient of cognitive control within the dorsomedial striatum. Neurolmage, 62(1), 41-47.

Ohlsson, S. (1992). Information-processing explanations of insight and related phenomena. Advances in the Psychology of Thinking, 1, 1-44.

Old, S. R., \& Naveh-Benjamin, M. (2008). Differential effects of age on item and associative measures of memory: A meta-analysis. Psychology and Aging, 23(1), 104-118.

Öllinger, M., Jones, G., \& Knoblich, G. (2008). Investigating theEffect of mental set on insight problem solving. Experimental Psychology, 55, 269-282.

Öllinger, M., Jones, G., \& Knoblich, G. (2014). The dynamics of search, impasse, and representational change provide a coherent explanation of difficulty in the nine-dot problem. Psychological Research, 78(2), 266-275.

Price, C. J. (2010). The anatomy of language: A review of $100 \mathrm{fMRI}$ studies published in 2009. Annals of the New York Academy of Sciences, 1191 (1), 62-88.

Ranganath, C., Cohen, M. X., Dam, C., \& D'Esposito, M. (2004). Inferior temporal, prefrontal, and hippocampal contributions to visual working memory maintenance and associative memory retrieval. Journal of Neuroscience, 24(16), 3917-3925.

Ridderinkhof, K. R., Ullsperger, M., Crone, E. A., \& Nieuwenhuis, S. (2004). The roleofthe medial frontal cortex in cognitivecontrol. Science, 306 (5695), 443-447.

Robertson, B. D., Hiebert, N. M., Seergobin, K. N., Owen, A. M., \& MacDonald, P. A. (2015). Dorsal striatum mediates cognitive control, not cognitive effort per se, in decision-making: An event-related fMRI study. Neurolmage, 114, 170-184.

Salvi, C., Bricolo, E., Franconeri, S. L., Kounios, J., \& Beeman, M. (2015). Sudden insight is associated with shutting out visual inputs. Psychonomic Bulletin \& Review, 22(6), 1814-1819.

Sandkühler, S., \& Bhattacharya, J. (2008). Deconstructing insight: EEG correlates of insightful problem solving. PLoS One, 3(1), e1459.

Schooler, J. W., \& Melcher, J. (1995). The ineffability of insight. In S. M. Smith, T. B. Ward, \& R. A. Finke (Eds.), The creative cognition approach (pp. 97-133). Cambridge, MA: The MIT Press.

Shafto, M. A., Burke, D. M., Stamatakis, E. A., Tam, P. P., \& Tyler, L. K. (2007). On thetip-of-the-tongue: Neural correlates of increased wordfinding failures in normal aging. Journal of Cognitive Neuroscience, 19 (12), 2060-2070.

Shen, W., Tong, Y., Li, F., Yuan, Y., Hommel, B., Liu, C., \& Luo, J. (2018). Tracking the neurodynamics of insight: a meta-analysis of neuroimaging studies. Biological psychology, 138, 189-198.
Singer, T., Critchley, H. D., \& Preuschoff, K. (2009). A common role of insula in feelings, empathy and uncertainty. Trends in Cognitive Sciences, 13(8), 334-340.

SR RESEARCH. (2007). Eyelink user manual. Version 1.3.0 [Computer software manual]. Ottawa, ON: Author.

Sridharan, D., Levitin, D. J., \& Menon, V. (2008). A critical role for the right fronto-insular cortex in switching between central-executive and default-mode networks. Proceedings of the National Academy of Sciences, 105(34), 12569-12574.

Subramaniam, K., Kounios, J., Parrish, T. B., \& Jung-Beeman, M. (2009). A brain mechanism for facilitation of insight by positive affect. Journal of Cognitive Neuroscience, 21(3), 415-432.

Tang, X., Pang, J., Nie, Q. Y., Conci, M., Luo, J., \& Luo, J. (2015). Probing the cognitive mechanism of mental representational change during chunk decomposition: A parametric fMRI study. Cerebral Cortex, 26(7), 2991-2999.

Thevenot, C., \& Oakhill, J. (2008). A generalization of the representational change theory from insight to non-insight problems: The case of arithmetic word problems. Acta Psychologica, 129, 315-324.

Tik, M., Sladky, R., Luft, C. D. B., Willinger, D., Hoffmann, A., Banissy, M. J., Windischberger, C. (2018). Ultra-high-field fMRI insights on insight: Neural correlates of the Aha!-moment (2018) Human Brain Mapping. https://doi.org/10.1002/hbm.24073.

Tonkonogy, J., \& Goodglass, H. (1981). Language function, foot of the third frontal gyrus, and rolandic operculum. Archives of Neurology, 38 (8), 486-490.

Topolinski, S., \& Reber, R. (2010). Gaining insight into the "Aha" experience. Current Directions in Psychological Science, 19(6), 402-405.

Tzourio-Mazoyer, N., Landeau, B., Papathanassiou, D., Crivello, F., Etard, O., Delcroix, N., ... Joliot, M. (2002). Automated anatomical labeling of activations in SPM using a macroscopic anatomical parcellation of the MNI MRI single-subject brain. Neurolmage, 15(1), 273-289.

Uddin, L. Q. (2015). Salience processing and insular cortical function and dysfunction. Nature Reviews Neuroscience, 16(1), 55.

Visser, M., \& Lambon Ralph, M. A. (2011). Differential contributions of bilateral ventral anterior temporal lobe and left anterior superior temporal gyrus to semantic processes. Journal of Cognitive Neuroscience, 23(10), 3121-3131.

Wu, L., Knoblich, G., \& Luo, J. (2013). The role of chunk tightness and chunk familiarity in problem solving: Evidence from ERPs and fMRI. Human Brain Mapping, 34(5), 1173-1186.

Zhao, Q., Zhou, Z., Xu, H., Chen, S., Xu, F., Fan, W., \& Han, L. (2013). Dynamic neural network of insight: $A$ functional magnetic resonance imaging study on solving Chinese 'chengyu' riddles. PloS One, 8(3), e59351.

How to cite this article: Becker M, Sommer T, Kühn S. Verbal insight revisited: $\mathrm{fMRI}$ evidence for early processing in bilateral insulae for solutions with AHA! experience shortly after trial onset. Hum Brain Mapp. 2020;41:30-45. https://doi. org/10.1002/hbm.24785 